\title{
Prediction-step staggered-in-time FDTD: An efficient numerical scheme to solve the linearised equations of fluid dynamics in outdoor sound propagation
}

\author{
T. Van Renterghem *, D. Botteldooren \\ Ghent University, Department of Information Technology, Sint-Pietersnieuwstraat 41, B-9000 Gent, Belgium
}

Received 23 December 2004; received in revised form 10 June 2005; accepted 7 July 2005

Available online 28 November 2005

\begin{abstract}
The finite-difference time-domain (FDTD) method to solve the linearised equations of fluid dynamics has shown to be very powerful and useful in outdoor sound propagation. Practical applications are however limited due to the large need for computational resources. The numerical discretisation influences computational efficiency to an important degree. In this paper, some possible ways to discretise temporal derivatives are studied. Two obvious ways of time-discretisation namely staggered-in-time (SIT) and a simple collocated-in-time (CIT) scheme are compared to the prediction-step staggered-in-time (PSIT) scheme. The latter is intended to be used for the calculation of sound propagation in the typical low wind speeds encountered in the outdoor environment at low heights above the earth's surface. It was shown that the PSIT scheme is more stable than the SIT scheme, so practical calculations are possible. Computational efficiency is increased to an important degree compared to the CIT scheme. The numerical accuracy (more precisely the amplitude error) of the PSIT scheme is an important improvement upon SIT. The CIT scheme on the other hand conserves amplitude better. The amplitude error becomes larger with increasing wind speed because of some simplifications during the numerical discretisation. In low wind speeds, the PSIT algorithm can serve as an interesting compromise between numerical accuracy and the required amount of computing power.
\end{abstract}

(c) 2005 Elsevier Ltd. All rights reserved.

\footnotetext{
* Corresponding author.

E-mail address: Timothy.Van.Renterghem@intec.ugent.be (T. Van Renterghem).
} 
Keywords: Finite-difference time-domain method; Linearised equations of fluid dynamics; Outdoor sound propagation; Numerical schemes

\section{Introduction}

The finite-difference time-domain (FDTD) technique to solve the linearised equations of fluid dynamics has become a reference model for complicated outdoor sound propagation calculations [1-4]. The main advantage upon other simulation techniques is the ability to take into account in detail arbitrary flow and temperature fields. This method has shown to be very useful to investigate practical problems, e.g., the use of trees as a curing measure for screen-induced refraction of sound by wind [2]. The FDTD method is however computational very expensive. The numerical discretisation plays an important role in this respect. In this paper, we will focus on explicit and compact (low-order) numerical schemes, in a Cartesian grid. Compact schemes are preferred for their easy inclusion of complicated obstacles and easy handling near interfaces between different propagation media (like the air-ground interface). Since the simulation space in outdoor sound propagation applications is usually very large, explicit schemes are interesting because they scale very well.

In a medium at rest, a staggered spatial grid and staggered time-discretisation results in a very efficient numerical scheme for solving the linear, scalar wave equation. Staggeredin-space means that the acoustical variables (pressure and the components of the particle velocity) are not discretised at the same physical locations in the computational grid. Staggered-in-time means that the pressure and velocity fields are not updated at the same, discrete times. Central difference approximations of the spatial and temporal derivatives in such a scheme result in second-order accuracy [5]. The scheme is very compact, which makes the implementation of boundary conditions simpler. In a staggered spatial grid, the acoustic pressures are typically situated in the centre of each computational cell, the components of the particle velocity are on the faces that border each cell. This means, e.g., that a rigid boundary condition is obtained by simply setting the (orthogonal) particle velocity at that location to zero.

The scheme is also very efficient in memory usage. Staggered-in-time allows for in-place computation: the new values of the acoustic pressure and acoustic velocity replace the old ones in computer memory. Since memory use is often the bottleneck in FDTD simulations, this is an important feature. Stability is ensured as long as the Courant number is smaller than or equal to 1 [5]. The numerical model is free of amplitude errors [5]. The phase error is smallest when propagating along the diagonal of the computational cells and if the Courant number is 1 [5].

The unique performance of such a scheme gets lost when the medium of propagation is moving. The spatial derivatives of the additional terms in the equations, related to the flow, must now be calculated over two cells. This does not create a problem: only a special treatment near the borders of the grid is needed. So a staggered-in-space scheme can still be used. The time-discretisation on the other hand is critical with respect to numerical stability. The field values required to calculate the additional terms induced by the flow are not available at the correct time and thus interpolation is required. Still using the staggered-in-time approach in combination with an explicit scheme in a moving medium 
results in stability issues, as will be shown in Section 4 in this paper. Calculations using this approach are nevertheless possible in some mild situations. A staggered-in-space, staggered-in-time scheme was used, e.g., to simulate the propagation of an acoustic pulse over short distances above a flat, rigid ground in the presence of small wind speeds and wind speed gradients [7]. In case of larger wind speeds, complex wind fields, and certainly when long simulation times are needed (e.g., in case of multiple reflections), instability will make meaningful simulations impossible.

The stability problem can be overcome by using a collocated temporal grid $[4,6]$. The acoustic pressure and the components of the particle velocity are updated in the latter at the same discrete times. In Section 4, it will be shown that such a scheme guarantees stability, even for Mach numbers up to 1. However, compared to the staggered-in-time scheme, the computational requirements increase to an important degree.

An alternative scheme, namely the "prediction-step staggered-in-time" (PSIT) algorithm [2,3], is studied in detail in this paper, and compared to the time-discretisation schemes mentioned in previous paragraphs. The PSIT scheme is a compromise between stability, accuracy and numerical efficiency. The main goal is the simulation of sound propagation outdoors in presence of the typical (low) wind speeds and wind speed gradients encountered at low heights above the earth's surface.

This paper is outlined as follows. The starting equations for sound propagation in a moving medium are given in Section 2. The PSIT algorithm is developed in Section 3. As an example, the time-discretisation of the mass-conservation equation is shown and the typical features of the PSIT scheme are discussed. The developed scheme is compared to a staggered-in-time and a collocated-in-time scheme in a two-dimensional simulation space with regard to numerical stability (Section 4) and accuracy (Section 5). Computational requirements of these numerical schemes are addressed in Section 6. Practical guidelines concerning numerical accuracy, computational effort and stability are summarized in Section 7. Finally, conclusions are drawn in Section 8.

\section{Moving-medium sound propagation equations}

Sound propagation in a moving medium can be described by the following set of linear equations $[1,3,6,7]$ :

$$
\begin{aligned}
& \frac{\partial p}{\partial t}+\mathbf{v}_{0} \cdot \nabla p+c^{2} \rho_{0} \nabla \cdot \mathbf{v}=0 \\
& \frac{\partial v}{\partial t}+\left(\mathbf{v}_{0} \cdot \nabla\right) \mathbf{v}+(\mathbf{v} \cdot \nabla) \mathbf{v}_{0}+\frac{1}{\rho_{0}} \nabla p=\mathbf{0},
\end{aligned}
$$

where $\mathbf{v}$ is the particle velocity vector, $p$ is the acoustic pressure, $\mathbf{v}_{0}$ is the background flow velocity vector, $c$ is the adiabatic speed of sound, $\rho_{0}$ is the ambient mass density, and $t$ is time. It is assumed that gravity is neglected, and that the background flow is incompressible.

The interactions between the sound waves and the flow are limited when using this set of equations. The acoustic waves do not influence the flow field, and generation of sound is not considered. The main effects of wind on sound propagation outdoors, namely convection, refraction and scattering are accounted for in detail. This set of equations describes sound propagation in a "background flow". A steady-state flow field is commonly used as an input for the transient acoustic calculations. Flow calculations can be performed 
independently from the acoustic calculations. This is an interesting approach, since both types of simulations have different demands concerning the computational grid and time steps. It is also possible to use commercial computational fluid dynamics (CFD) software, which makes use of state-of-the-art numerical schemes for flow calculations, suitable turbulence models, etc.

\section{PSIT algorithm}

\subsection{Notations}

In this paper, focus is on time-discretisation schemes. All the schemes considered use a staggered-in-space grid. The same notations as in [5] are used. Indices of spatial and temporal grid points are indicated as subscripts and superscripts, respectively. The acoustic pressures are determined at grid positions $(i \mathrm{~d} x, j \mathrm{~d} y, k \mathrm{~d} z)$ at sampled times $l \mathrm{~d} t$ :

$$
p_{(i \mathrm{~d} x, j \mathrm{~d} y, k \mathrm{~d} z)}^{l \mathrm{~d} t},
$$

where $\mathrm{d} x, \mathrm{~d} y$, and $\mathrm{d} z$ are the spatial discretisation steps and $\mathrm{d} t$ is the time discretisation step. The indices $i, j$ and $k$ locate the spatial points, the index $l$ indicates a discrete time.

The three components of the particle velocity are determined at staggered grid positions

$$
\mathrm{v}_{x((i \pm 0.5) \mathrm{d} x, j \mathrm{~d} y, k \mathrm{~d} z)}^{(l+0.5 s) \mathrm{d} t}, \quad \mathrm{v}_{y(i \mathrm{~d} x,(j \pm 0.5) \mathrm{d} y, k \mathrm{~d} z)}^{(l+0.5 s)}, \quad \mathrm{v}_{z(i \mathrm{~d} x, j \mathrm{~d} y,(k \pm 0.5) \mathrm{d} z)}^{(l+0.5) \mathrm{d} t} .
$$

In case of a staggered-in-time discretisation, $s$ equals 1 . This means that the particle velocities are updated at intermediate times. When the time-discretisation is collocated, $s$ equals 0 . In the latter, the velocity is updated at the same discrete times as the pressures.

The (time-independent) components of the background flow velocity can be discretised at the same positions of the particle velocity components:

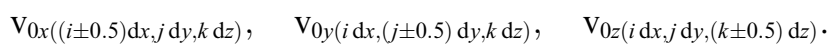

\subsection{SIT and CIT}

To illustrate the differences in discretisation of the schemes that are considered, the pressure equation (1) is studied in detail. The following equations are continuous in space and discrete in time. Some possibilities for the time-discretisation of the sound propagation equations in a moving medium are explained briefly in this section.

In a first approach, the staggered-in-time method is used for the time-discretisation of the non-moving medium terms, while the term containing the background flow velocity is approximated at the time $(l-1) \mathrm{d} t[7]$ :

$$
p^{l}=p^{l-1}-\mathrm{d} t c^{2} \rho_{0} \nabla \cdot \mathbf{v}^{l-0.5}-\mathrm{d} t \mathbf{v}_{0} \cdot \nabla p^{l-1} .
$$

In this way, an explicit time-stepping algorithm is obtained: the pressure at the new time $l \mathrm{~d} t$ is based completely upon values of the previous times $(l-1) \mathrm{d} t$ and $(l-0.5) \mathrm{d} t$. Inplace computation is possible. Additional memory, compared to the equations that describe sound propagation in a medium at rest, is only needed to store values of the background flow velocity. Since Eq. (6) is closely related to the staggered-in-time approach in absence of flow, it will be indicated throughout this paper as SIT. 
Alternatively both the acoustic pressure and acoustic velocity components can be discretised at times $l \mathrm{~d} t$. The time derivative can now be approximated by a central difference over 2 time steps and gives:

$$
p^{l}=p^{l-2}-2 \mathrm{~d} t c^{2} \rho_{0} \nabla \cdot \mathbf{v}^{l-1}-2 \mathrm{~d} t \mathbf{v}_{0} \cdot \nabla p^{l-1} .
$$

Eq. (7) is the simplest form of a collocated-in-time scheme, and will be indicated throughout this paper as CIT. The values of the acoustic pressure of two previous times $(l-2) \mathrm{d} t$ and $(l-1) \mathrm{d} t$ are now needed to calculate the acoustic pressure at the new time $l \mathrm{~d} t$. This means that the memory requirement is doubled compared to time-stepping based on Eq. (6).

\subsection{PSIT}

The same temporal grid is used as in the SIT approach ( $s$ equals 1 in Eq. (4)). The term containing the background flow velocity is now taken as half the value at the previous time $(l-1) \mathrm{d} t$, and half the value at time $l \mathrm{~d} t$ :

$$
p^{l}=p^{l-1}-\mathrm{d} t c^{2} \rho_{0} \nabla \cdot \mathbf{v}^{l-0.5}-0.5 \mathrm{~d} t \mathbf{v}_{0} \cdot \nabla p^{l}-0.5 \mathrm{~d} t \mathbf{v}_{0} \cdot \nabla p^{l-1} .
$$

Such an approach improves stability, as will be shown in Section 4. Writing the unknown quantities at the left hand side gives:

$$
p^{l}+0.5 \mathrm{~d} t \mathbf{v}_{0} \cdot \nabla p^{l}=p^{l-1}-\mathrm{d} t c^{2} \rho_{0} \nabla \cdot \mathbf{v}^{l-0.5}-0.5 \mathrm{~d} t \mathbf{v}_{0} \cdot \nabla p^{l-1} .
$$

For the discretisation of the spatial derivatives at time $l \mathrm{~d} t$, neighboring values of $p$ are involved. The numerical scheme is no longer explicit. This would lead to a band matrix to be inverted at each time step. Since we are only interested in sound propagation in the typical (low) wind speeds encountered outdoors at low heights above the earth's surface, an alternative and more efficient approach is chosen. This allows approximating the gradient of the pressure at time $l \mathrm{~d} t$ by neglecting the background flow:

$$
p_{\text {appr. }}^{l} \approx p^{l-1}-\mathrm{d} t c^{2} \rho_{0} \nabla \cdot \mathbf{v}^{l-0.5} .
$$

In this approach, following terms on the left-hand side in Eq. (9) are neglected:

$$
-0.25 \mathrm{~d} t^{2} \mathbf{v}_{0} \cdot \nabla\left[\mathbf{v}_{0} \cdot \nabla p^{l-1}\right] \text { and }-0.25 \mathrm{~d} t^{2} \mathbf{v}_{0} \cdot \nabla\left[\mathbf{v}_{0} \cdot \nabla p^{l}\right],
$$

that are second-order terms in Mach number.

Using Eq. (10) in (9) gives:

$$
p^{l}=p^{l-1}-\mathrm{d} t c^{2} \rho_{0} \nabla \cdot \mathbf{v}^{l-0.5}-\mathrm{d} t \mathbf{v}_{0} \cdot \nabla\left(p^{l-1}-0.5 \mathrm{~d} t c^{2} \rho_{0} \nabla \cdot \mathbf{v}^{l-0.5}\right) .
$$

The part in between brackets on the right hand side can be seen as the sound propagation equation to calculate the pressure at time $(l-0.5) \mathrm{d} t$ in a medium at rest:

$$
p^{l}=p^{l-1}-\mathrm{d} t c^{2} \rho_{0} \nabla \cdot \mathbf{v}^{l-0.5}-\mathrm{d} t \mathbf{v}_{0} \cdot \nabla p_{\text {noflow }}^{l-0.5} .
$$

During the time-stepping algorithm, we have to make a "prediction" of the sound field as if there was no flow. This is done after finishing the calculation of the velocity field at time $(l-0.5) \mathrm{d} t$ and just before the new pressure field is calculated at time $l \mathrm{~d} t$.

As can be seen from Eq. (13), the value of $p$ at the new time $l \mathrm{~d} t$ only depends upon values at the previous times $(l-1) \mathrm{d} t$ and $(l-0.5) \mathrm{d} t$.

Actually, this equation is quite similar to the CIT Eq. (7). The second and third term on the right hand side are discretised at an intermediate time $(l-0.5) \mathrm{d} t$ in Eq. $(13),(l-1) \mathrm{d} t$ 
in Eq. (7) relative to the times necessary to discretise the temporal derivative of the pressure $(l \mathrm{~d} t$ and $(l-1) \mathrm{d} t$ in Eq. (13), $l \mathrm{~d} t$ and $(l-2) \mathrm{d} t$ in Eq. (7)). The main differences lie in the fact that the PSIT approach is more efficient in memory use (see Section 6), and that the CIT scheme does not use a low-Mach number approach.

The discretisation of the velocity Eq. (2) is based on this same approach, and will therefore not be discussed here. The time-discretised velocity equation, using the predictionstep staggered-in-time approach, yields:

$$
\mathbf{v}^{l+0.5}=\mathbf{v}^{l-0.5}-\mathrm{d} t \frac{1}{\rho_{0}} \nabla p^{l}-\mathrm{d} t\left(\mathbf{v}_{0} \cdot \nabla\right) \mathbf{v}_{\text {noflow }}^{l}-\mathrm{d} t\left(\mathbf{v}_{\text {noflow }}^{l} \cdot \nabla\right) \mathbf{v}_{0} .
$$

\section{Stability}

\subsection{Methodology}

Numerical stability of the different approaches considered to solve the moving-medium sound propagation equations is examined by using theory of discrete time-delay systems [8]. The spatial discretisation results in a number of local values for the acoustic pressures $p$ and components of the particle velocities $V_{i}$. A system matrix is constructed, which couples each acoustical variable to the relevant quantities at the previous time step(s).

For the matrices of unknowns to be finite, the simulation region of interest must be bounded. Although boundary conditions can influence stability to an important degree, they are not of interest in an assessment of stability of a bulk numerical scheme. Therefore, the grid considered will be periodically extended to an infinite area. The use of so-called cyclic boundary conditions is a straightforward implementation in a structured grid. Index values beyond the last element refer to the first elements in each direction and the other way around.

Let $P$ be the array containing all pressures, and $V_{i}$ the array containing the $i$-components of the particle velocities. The distinction is made between collocated-in-time schemes and staggered-in-time schemes. In the first case, Eqs. (1) and (2) can be written as

$$
\left\{\begin{array}{l}
M P^{l}=M_{-2} P^{l-2}+M_{-1} P^{l-1}+\sum_{i} R_{i} V_{i}^{l-1}, \\
N_{i} V_{i}^{l}=N_{i,-2} V_{i}^{l-2}+N_{i,-1} V_{i}^{l-1}+S_{i} P^{l-1} .
\end{array}\right.
$$

In the second case, these equations become

$$
\left\{\begin{array}{l}
M P^{l}=M_{-1} P^{l-1}+\sum_{i} R_{i} V_{i}^{l-0.5}, \\
N_{i} V_{i}^{l-0.5}=N_{i,-1.5} V_{i}^{l-1.5}+S_{i} P^{l-1} .
\end{array}\right.
$$

In the previous equations $M, N, S$ and $R$ are sparse matrices containing the parameters belonging to the different terms in the sound propagation equations. To write these equations as a general matrix equation describing a discrete time-delay system $X^{m+1}=A X^{m}$, we find in case of Eq. (15):

$$
X^{l}=\left[\begin{array}{c}
P^{l} \\
V_{i}^{l} \\
P^{l-1} \\
V_{i}^{l-1}
\end{array}\right], \quad A=\left[\begin{array}{cccc}
M^{-1} M_{-1} & M^{-1} R_{i} & M^{-1} M_{-2} & 0 \\
N_{i}^{-1} S_{i} & N_{i}^{-1} N_{i,-1} & 0 & N_{i}^{-1} N_{i,-2} \\
1 & 0 & 0 & 0 \\
0 & 1 & 0 & 0
\end{array}\right]
$$


and in case of Eq. (16):

$$
X^{l}=\left[\begin{array}{c}
P^{l} \\
V_{i}^{l-0.5}
\end{array}\right], \quad A=\left[\begin{array}{cc}
M^{-1} M_{-1}+M^{-1} R_{i} N_{i}^{-1} S_{i} & M^{-1} R_{i} N_{i}^{-1} N_{r, i-1.5} \\
N_{i}^{-1} S_{i} & N_{i}^{-1} N_{i,-1.5}
\end{array}\right],
$$

where the index $i$ implicitly refers to an extension to as much vector-components as required and 0 and 1 indicate matrices of zeros and the unit matrix of appropriate dimensions.

It can be proven that the discrete system is stable if and only if all eigenvalues (or poles) of the matrix $A$ have a modulus smaller than one [8]. In other words, all eigenvalues must lie within a circle of radius 1 in the complex plane. The position of the eigenvalues gives an indication of the severity and type of instability. Besides, some aspects of the numerical accuracy become visible.

\subsection{Stability analysis}

A two-dimensional simulation space was considered, in case of a uniform background flow and in case of the presence of gradients in the background flow velocity. A grid of $10 \times 10$ cells (periodically extended) is used, leading to 300 eigenvalues for the SIT/PSIT system and 600 eigenvalues for the CIT system. All schemes use the staggered-in-space approach.

An explicit integration scheme in a staggered-in-space and staggered-in-time grid, in a non-moving medium, is stable when the Courant number is equal to or smaller than 1. This condition can be interpreted as follows: within a single time step, the acoustic wave may travel at most the distance of one computational cell. In this view, the Courant number for sound propagation in a uniform flow may be defined as:

$$
C N=\frac{\left(c+v_{0}\right) \mathrm{d} t}{\sqrt{\frac{1}{\mathrm{~d} x^{2}}+\frac{1}{\mathrm{~d} y^{2}}}}
$$

where $C N$ is the Courant Number, $c$ is the speed of sound, and $\mathrm{d} x$ and $\mathrm{d} y$ are the spatial discretisation steps in the two-dimensional grid, $\mathrm{d} t$ is the temporal discretisation step, and $v_{0}$ is the uniform flow velocity.

It was observed that the presence of gradients in the background flow does not significantly change the stability of the different numerical schemes considered. Stability is dominated by the maximum magnitude of the flow velocity in the computational grid. Therefore, the stability analysis in the remainder of this section is performed for the case of a uniform flow.

The results of the stability analysis are shown in Figs. 1 and 2. A uniform flow velocity of $10 \mathrm{~m} / \mathrm{s}$ (Mach number of 0.0294 ) is used. The poles are plotted in the complex plane. Using the CIT approach, a completely stable system is obtained, provided that the Courant number, as defined by Eq. (19), is equal to or smaller than 0.5. This alternative stability condition is caused by time-stepping over two times $\mathrm{d} t$ in the CIT equations. When this condition is fulfilled, all poles lie exactly on the unit circle, as shown in Fig. 1.

In case of a staggered-in-time scheme in the presence of flow, there are always some poles that lie outside the unit circle. The location of the poles in Fig. 2 is the result of the stability analysis in case of a Courant number, as defined by Eq. (19), equal to 1. 


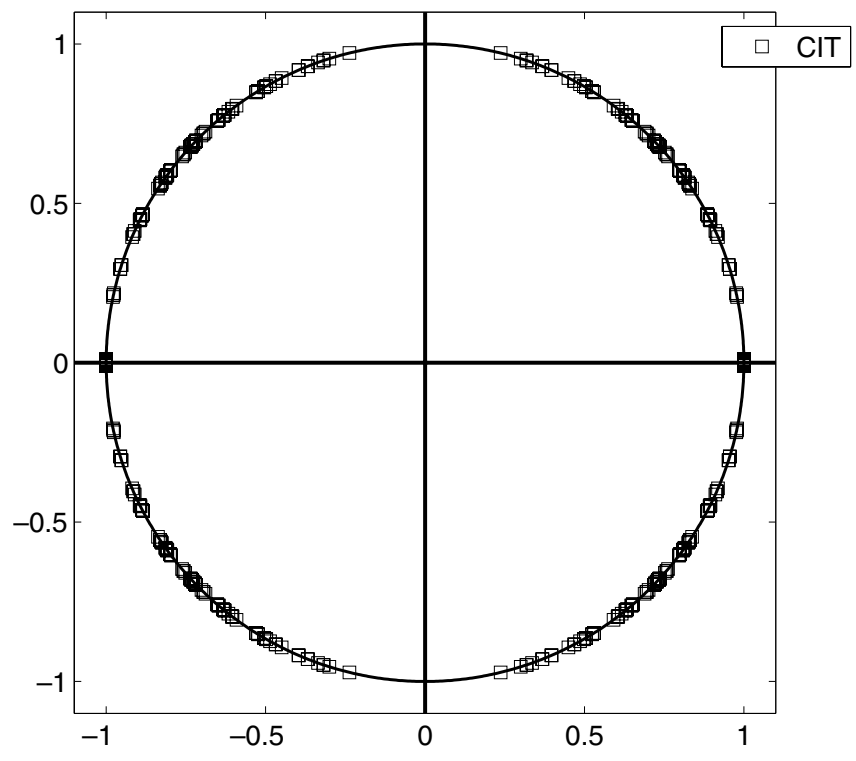

Fig. 1. Locations of the eigenvalues of the system matrix $A$ in the complex plane, for the CIT scheme. The uniform background flow velocity is $10 \mathrm{~m} / \mathrm{s}$, the Courant number equals 0.5 .

The distance between the unstable pole and the unit circle is an indication for the severity of the instability. Or stated otherwise: the larger this distance, the quicker the instability appears in a simulation. In Fig. 3, the maximum magnitude of the poles is shown for different Courant Numbers, as defined by Eq. (19), for a uniform flow velocity of $10 \mathrm{~m} / \mathrm{s}$ (Mach number of 0.0294). When decreasing the Courant number, there is a shift of the unstable poles towards the unit circle, however the instability (which becomes eventually very weak) remains. It can therefore be concluded that SIT and PSIT are theoretically unstable.

When comparing the PSIT and SIT scheme, it is clear that the PSIT algorithm is beneficial as to stability, since the poles lie more closely to the unit circle. When increasing the flow velocity (see Fig. 4), the poles shift outside the unit circle, and instability increases. It can be seen, e.g., that a flow velocity of $5 \mathrm{~m} / \mathrm{s}$ (Mach number of 0.0147 ) in the SIT scheme gives the same "severity of instability" at about $22 \mathrm{~m} / \mathrm{s}$ (Mach number of 0.0647 ) in the PSIT scheme. A Courant Number equal to 1, as defined by Eq. (19), is used for the PSIT/SIT schemes, and a Courant Number equal to 0.5, as defined by Eq. (19), for the CIT scheme.

Note that this analysis is performed for an infinitely extended open area. Boundary conditions like (perfectly) absorbing boundaries or bulk losses may stabilize the system.

\section{Numerical accuracy}

\subsection{Uniform flow}

The numerical accuracy of the different time-discretisation schemes is studied in a two-dimensional simulation space. Exact analytical solutions for sound propagation in 

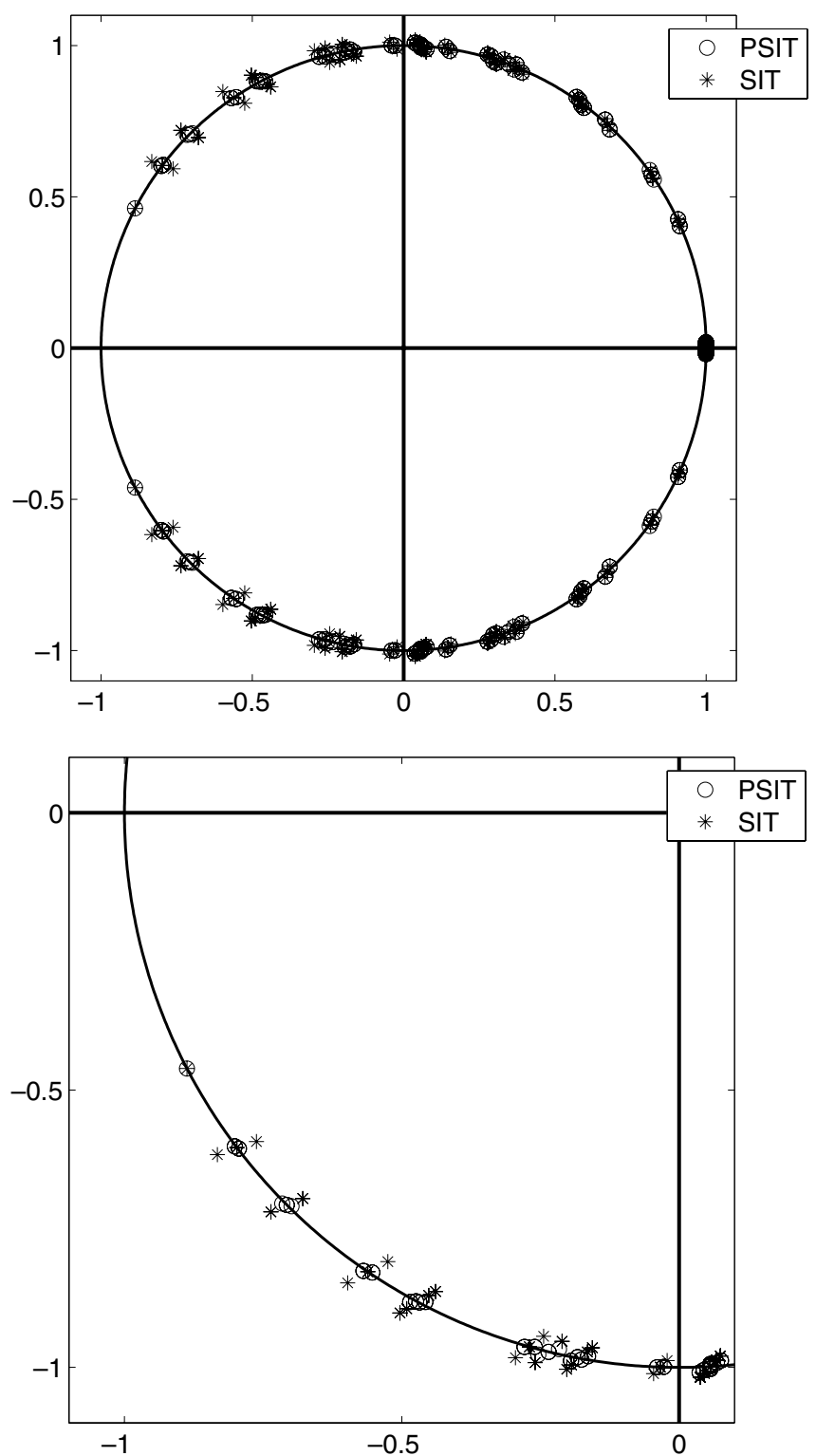

Fig. 2. Locations of the eigenvalues of the system matrix $A$ in the complex plane, for the SIT and PSIT scheme. The uniform background flow velocity is $10 \mathrm{~m} / \mathrm{s}$, the Courant number equals 1 . In the figure above, a general view is given; in the figure below, a single quadrant is shown in detail.

a moving medium are limited to the case of a uniform flow. Therefore, the numerical accuracy of the different schemes that are considered in this paper is first studied in case of a uniform flow velocity of $10 \mathrm{~m} / \mathrm{s}$ (Mach number of 0.0294). A formula to calculate the sound field due to a point monochromatic source in a two-dimensional homogeneous uniformly moving medium is given in [6]. Although this rather simple situation, the differences in accuracy between the schemes under consideration become clear. 


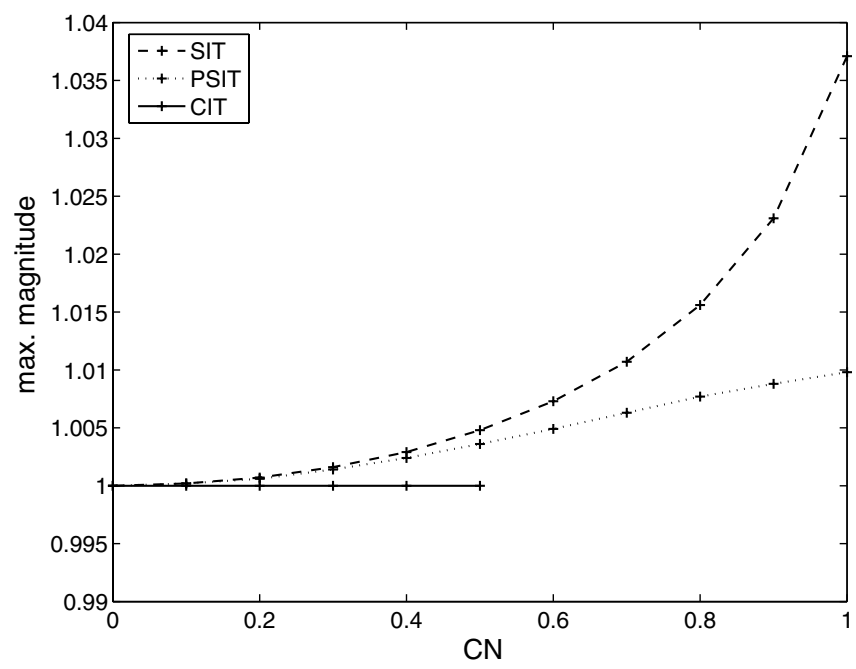

Fig. 3. Maximum magnitude of the eigenvalues for the SIT, PSIT and CIT scheme, with increasing Courant number. The uniform background flow velocity equals $10 \mathrm{~m} / \mathrm{s}$.

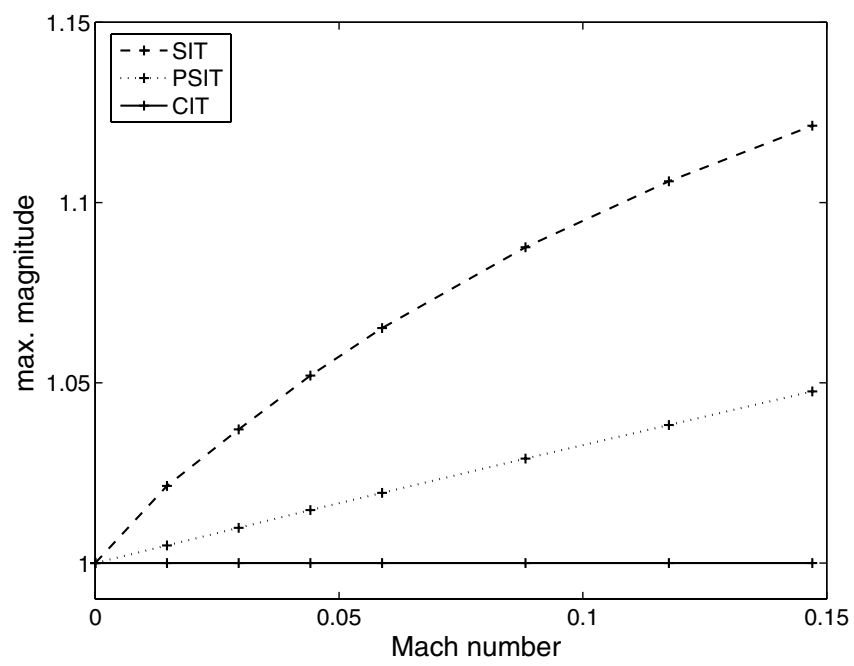

Fig. 4. Maximum magnitude of the eigenvalues for the $\operatorname{SIT}(C N=1)$, PSIT $(C N=1)$ and CIT $(C N=0.5)$ scheme, with increasing uniform background flow Mach number.

All schemes use a staggered, spatial grid. A point source emits an acoustic pulse. A point source in a two-dimensional grid is equivalent to an infinite, coherent line source in a three-dimensional space. The numerical accuracy of a FDTD scheme is characterized by its amplitude and phase error. Therefore, at two locations (point $p_{1}$ and point $p_{2}$ ), close to each other, time signals are recorded. The ratio in amplitude between these two points as calculated with FDTD is compared to the ratio that can be expected theoretically. The phase difference as predicted by FDTD, subtracted from the theoretical phase difference, 
gives the phase error. The amplitude error is expressed in $\mathrm{dB}$ per metre to have an estimation of the accuracy in practical calculations. The phase error is expressed in radials per metre. In outdoor sound propagation, a small amplitude error is of primary concern. When resonant structures are present, a phase error close to 0 becomes important.

In Fig. 5, accuracy is presented as a function of the wavelength $\lambda$ divided by the spatial discretisation step $\mathrm{d} x$. The Courant number, as defined by Eq. (19), equals 1 for the SIT and PSIT scheme, and equals 0.5 for the CIT scheme. The flow is directed along one axis of the grid $\left(0^{\circ}\right)$. Three situations are considered: sound propagation along one axis in direction of the flow $\left(0^{\circ}\right)$, sound propagation along the diagonal of the (square) cells $\left(45^{\circ}\right)$ and sound propagation along the other axis of the grid, thus orthogonal to the flow $\left(90^{\circ}\right)$. The results of the SIT, PSIT and CIT calculations are shown. For comparison, the results of the SIT calculations in absence of flow are added.

In absence of flow, there is no amplitude error in the SIT approach. For sound propagation along the diagonal of the computational cells, the phase error is very small. The latter increases when deviating from that (optimal) direction, and reaches its maximum
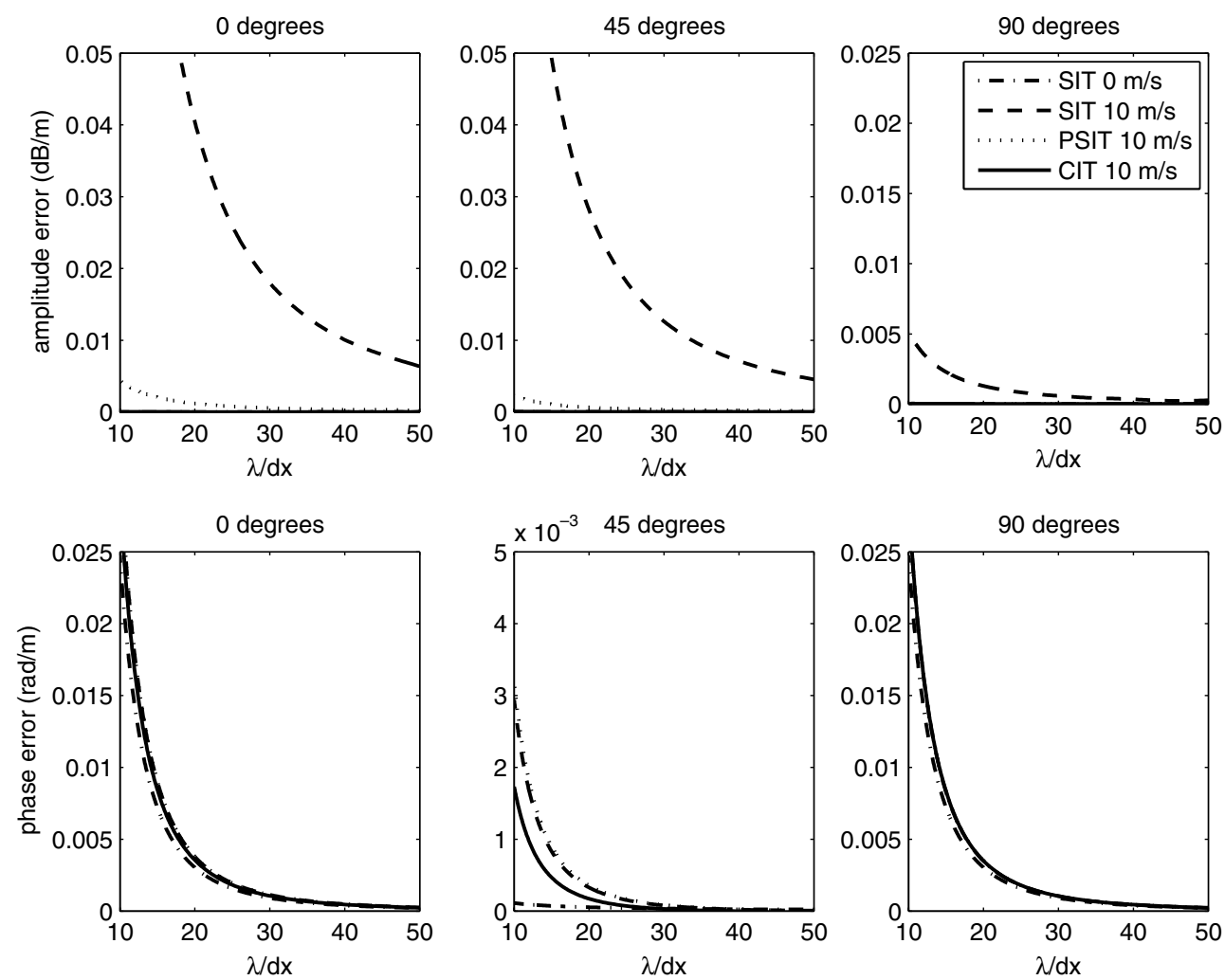

Fig. 5. Amplitude error (figures above) and phase error (figures below) with increasing number of cells per wavelength, for three different propagation conditions. The SIT $(C N=1)$, PSIT $(C N=1)$ and $C I T(C N=0.5)$ approach in case of a uniform background flow velocity of $10 \mathrm{~m} / \mathrm{s}$ are considered. For comparison, the numerical accuracy of the SIT approach in a non-moving medium $(C N=1)$ is also shown. In the upper plots, the lines indicating the latter are covered up by the lines of the CIT scheme. 
for sound propagation along one dimension. With an increasing number of cells per wavelength, the phase error decreases. These findings were shown analytically in [5]. The amplitude error and phase error of the CIT approach in flow follows closely the behavior of the SIT scheme in a non-moving medium, except for the phase error when propagating along the diagonal of the cells.

The amplitude of the SIT and PSIT scheme in flow is not error-free. When using 10 computational cells per wavelength in case of a uniform flow velocity of $10 \mathrm{~m} / \mathrm{s}$, the SIT scheme gives an amplitude error of $0.15,0.1$ and $5 \mathrm{E}-3 \mathrm{~dB} / \mathrm{m}$, for sound propagation in the direction of the flow $\left(0^{\circ}\right)$, along the diagonal of the computational cells $\left(45^{\circ}\right)$ and orthogonal to the flow $\left(90^{\circ}\right)$, respectively. Using the PSIT approach, these errors become $4 \mathrm{E}-3,2 \mathrm{E}-3$ and $<1 \mathrm{E}-4 \mathrm{~dB} / \mathrm{m}$. When applying the CIT scheme or the SIT scheme in absence of flow with this same spatial discretisation, the amplitude error was found to be smaller than $1 \mathrm{E}-4 \mathrm{~dB} / \mathrm{m}$ in the directions that were examined. It should be mentioned that the values given for the SIT scheme are extrapolated ones, neglecting possible instability.

It can be observed that the amplitude error for PSIT and SIT are smaller for propagation along the diagonal of the cells. For sound propagation orthogonal to the flow, the amplitude errors of all schemes are very small, except for the SIT scheme.

The PSIT scheme has clearly a much smaller amplitude error than the SIT scheme. The stability analysis already revealed this. There is no amplitude error when the poles of the system lie exactly on the unit circle in the complex plane, as is the case for the CIT scheme. It is clear from Figs. 2 and 6 that the stable poles (those inside the unit circle) of the SIT scheme have a smaller modulus than the stable PSIT poles.

The amplitude error in the SIT scheme is caused by the fact that the flow terms are discretised at the "wrong time". In the PSIT scheme, the amplitude error is caused by neglecting the flow during the prediction step. The minimum value of the magnitude of all poles in the system, which is an indication for the amplitude error, with increasing flow velocity, is shown in Fig. 6. With increasing flow velocity, this error increases.

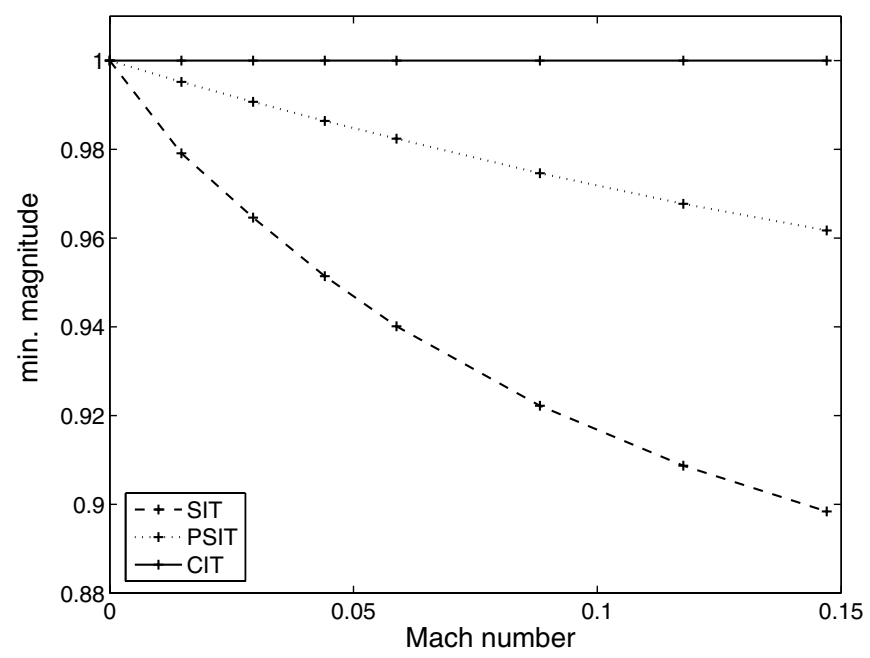

Fig. 6. Minimum magnitude of the eigenvalues for the $\operatorname{SIT}(C N=1)$, PSIT $(C N=1)$ and $C I T(C N=0.5)$ scheme, with increasing uniform background flow Mach number. 
Introducing flow only slightly affects the phase error. The phase is not anymore errorfree when propagating along the diagonal of the cells, since the flow is directed along one axis of the grid. The different schemes in flow behave more or less similarly.

\subsection{Non-uniform flow}

Numerical accuracy is further investigated in case of a non-uniform flow. Since no exact analytical solutions are known in such a sound propagation medium, the CIT approach is chosen as the reference solution. As was shown by the accuracy study in case of a uniform flow, the amplitude error was very small for the latter. This approach does however not allow investigating the phase error. An artificial sawtooth wind speed profile was chosen. Wind speed is directed horizontally. A linear wind speed gradient of $1 / \mathrm{s}$ is used between the maxima $(10 \mathrm{~m} / \mathrm{s})$ and minima $(0 \mathrm{~m} / \mathrm{s})$ in this profile. Such a profile contains upwardly and downwardly refracting parts. The source is situated at a height were a maximum in the wind speed is present. Downwind sound propagation is simulated in the free field, in horizontal direction, up to a distance of $100 \mathrm{~m}$ from the source.

Calculations with the SIT approach were not possible since instabilities arose before the receiver at $100 \mathrm{~m}$ was reached. Calculations with the PSIT scheme were sufficiently stable. The accuracy of the latter, compared to the CIT approach, is calculated in our example. When taking 10 computational cells per wavelength, the amplitude error becomes $0.5 \mathrm{~dB}$ at a distance of $100 \mathrm{~m}$. This means that the increase in the error, compared to the uniform flow case (with a wind speed equal to $10 \mathrm{~m} / \mathrm{s}$ ), is only $1 \mathrm{E}-3 \mathrm{~dB} / \mathrm{m}$. It can therefore be concluded that numerical accuracy is mainly determined by the magnitude of the flow velocity. The error induced by the presence of gradients in the flow velocity is of secondary importance.

\section{Computational efficiency}

In a (homogeneous) medium at rest, memory requirements are mainly determined by the number of acoustical variables $p$ and $v_{i}$. The SIT approach in absence of flow is computational very efficient and is therefore taken as a reference. Such a scheme allows for inplace computation: the new values of both the acoustic pressures and the components of the particle velocities replace the old values in computer memory.

In a moving medium, the (background) flow velocity field needs to be stored as well. In case of a uniform flow, the number of additional variables is only limited to the number of dimensions of the grid. In the most general situation, and when discretising the components of the background flow velocities at the locations of the components of the particle velocities (see Section 3.1), the fields of $v_{0 i}$ need to be stored and use the same amount of memory as the $v_{i}$ fields.

The SIT approach in flow, compared to the SIT scheme in a medium at rest, only needs additional memory to store values of the background flow velocity. For the CIT scheme, the values of the acoustical variables of two time steps need to be kept in memory as well, leading to a doubling of memory use when comparing with the SIT scheme in flow.

It might seem that for the PSIT scheme the same amount of memory is needed as for the CIT scheme. However, when using the same memory locations to temporarily store the predictions of the pressures and the predictions of the components of the velocities, memory use is increased to a lesser degree. In Table 1, an overview is given of the number of 
Table 1

Number of acoustical variables $p$ and $v_{i}$ needed in the SIT, PSIT and CIT scheme, in case of a one-dimensional, two-dimensional and three-dimensional simulation space

\begin{tabular}{llll}
\hline & SIT & PSIT & CIT \\
\hline $1 \mathrm{D}$ & 2 & 3 & 4 \\
2D & 3 & 4 & 6 \\
$3 \mathrm{D}$ & 4 & 5 & 8 \\
\hline
\end{tabular}

acoustical variables $p$ and $v_{i}$ needed to perform simulations in a one-dimensional, twodimensional and three-dimensional simulation space. The memory needed to store values of the background flow velocity is not considered in Table 1 .

In a two-dimensional grid, the memory requirements of the PSIT scheme are $2 / 3$ of those of the CIT scheme. In a three-dimensional simulation space, this ratio reduces further to 5/8. It is clear that the PSIT approach is beneficial as to memory use.

The different schemes can be ordered with regard to computation speed as follows: SIT $>$ PSIT $>$ CIT. The SIT and PSIT scheme perform time-stepping with the same temporal discretisation step, leading to a Courant Number equal to 1. An additional calculation is needed at each time step in the PSIT scheme, for each acoustical variable. This results in an important increase in calculation time of PSIT compared to SIT. Computing times are however less than doubled, since during the prediction step the background flow is neglected. This means that fewer terms need to be calculated.

The CIT scheme is slower, since the Courant Number has to be limited to 0.5, resulting in a doubling of the number of time steps needed. Besides, at each time step, all flow terms in the discretised equation need to be included.

\section{Discussion}

The SIT and PSIT scheme are computational much more efficient than the CIT scheme. On the other hand, it is clear the CIT approach is better compared to PSIT and certainly to SIT as regards numerical accuracy. The applicability of the SIT scheme seems doubtful because of the large amplitude error and numerical instability. Calculations with the SIT scheme in [7] were however in good agreement with analytical solutions. It has to be mentioned that wind speeds were very low in these calculations, in combination with very short propagation distances. Besides, almost 20 computational cells were used for the highest frequency under consideration.

When using the PSIT scheme for downwind sound propagation in a uniform flow of $10 \mathrm{~m} / \mathrm{s}$, only $0.4 \mathrm{~dB}$ is numerically dissipated over a distance of $100 \mathrm{~m}$ and when using 10 computational cells per wavelength. The presence of gradients in the flow velocity only slightly increases this error. In practical applications, when using a broadband source, this will be the maximum error, and only applies to the highest frequency that is modeled. The increased accuracy that can be obtained with the CIT scheme must be weighted against the increase in computational effort when performing calculations. Sound propagation calculations over long distances need very large amounts of computational resources, even with a scheme like PSIT.

Examples of the usefulness of the PSIT scheme in non-uniform flows can be found in [2]. Numerical calculations are compared to measurements in two wind tunnel experiments with noise barriers, described in detail in [9-11]. Flow fields in the latter are complex 
because of the presence of large gradients in the flow velocity and also by the presence of rotating flows. The propagation distances in these experiments were smaller than $100 \mathrm{~m}$. In a first one [9], sound propagation over a single noise barrier in flow was modeled. Simulations with the PSIT [2] approach were shown to be accurate. In a second experiment, noise barriers on both sides of a source were modeled, in combination with wind speed reducing measures $[10,11]$. The flow fields near the barriers consisted in both upwardly and downwardly refracting flows. Because of the multiple reflections between the barriers, time signals were long. It was found that SIT simulations were not possible in such a configuration, not only for the low accuracy of this scheme, but also because instability interfered with the relevant signals, preventing meaningful simulations. The use of the PSIT approach resulted in sufficiently stable calculations. Good agreement was obtained with experimental results [2]. About 10 computational cells per wavelength were used for these calculations (for sound propagation in air), for the highest frequencies considered. The maximum magnitude of the wind velocity in the simulation grids was in all cases smaller than $20 \mathrm{~m} / \mathrm{s}$.

\section{Conclusions}

In this paper, the prediction-step staggered-in-time (PSIT) finite-difference timedomain scheme is studied in detail. Focus was on the time-discretisation of the moving-medium sound propagation equations, for compact and explicit numerical schemes in a Cartesian grid. A comparison with more obvious time-discretisation schemes like staggered-in-time (SIT) and collocated-in-time (CIT) was performed. It was shown that the PSIT scheme is a good compromise between these schemes. In contrast to the SIT scheme, stability is improved to an important degree, so the simulation of sound propagation in the outdoor environment in the presence of realistic wind fields near the earth's surface becomes possible. Compared to the CIT scheme, computational efficiency is strongly increased, as concerns both computing times and memory use. The phase error of all schemes considered is very similar. The amplitude error of the PSIT scheme is much smaller than the amplitude error of the SIT scheme. In the CIT approach however, there is in theory no amplitude error. It was found that the magnitude of the flow velocity is dominant to the presence of gradients, as concerns both stability and numerical accuracy.

With increasing wind speed, the amplitude error increases in the PSIT algorithm. This is caused by the prediction step where the background flow is neglected in order to have an explicit and efficient numerical scheme. Depending on the available computational resources, the magnitude of the wind speed and the desired accuracy, an appropriate time-discretisation scheme should be used. The PSIT algorithm is a compromise between numerical accuracy, computing time and memory use.

\section{References}

[1] Blumrich R, Heimann D. A linearized Eulerian sound propagation model for studies of complex meteorological effects. J Acoust Soc Am 2002;112:446-55.

[2] Van Renterghem T, Botteldooren D. Numerical simulation of the effect of trees on downwind noise barrier performance. Acta Acust Acust 2003;89:764-78.

[3] Van Renterghem T. The finite-difference time-domain method for the simulation of sound propagation in a moving medium. PhD thesis, Universiteit Gent, Belgium; 2003.. 
[4] Wilson DK, Moran ML, Liu L, Ostashev VE, Aldridge DF, Symons NP, et al. Development of a highfidelity simulation capability for battlefield acoustics. In: Proceedings of SPIE aerosense, Orlando (FL, USA); 2003..

[5] Botteldooren D. Finite-difference time-domain simulation of low-frequency room acoustic problems. J Acoust Soc Am 1995;98:3302-8.

[6] Ostashev VE, Wilson DK, Liu L, Aldridge DF, Symons NP, Marlin D. Equations for finite-difference, timedomain simulation of sound propagation in moving inhomogeneous media and numerical implementation. $\mathbf{J}$ Acoust Soc Am 2005;117:503-17.

[7] Salomons EM, Blumrich R, Heimann D. Eulerian time-domain model for sound propagation over a finiteimpedance ground surface. Comparison with frequency-domain models. Acta Acust Acust 2002;88:483-92.

[8] Debeljkovic DL, Aleksendric M, Yi-Yong N, Zhang QL. Lyapunov and non-Lyapunov stability of linear discrete time delay systems. Facta universitatis, series: mechanical engineering 2002;1:1147-60.

[9] Salomons EM. Reduction of the performance of a noise screen due to screen-induced wind-speed gradients. Numerical computations and wind tunnel experiments. J Acoust Soc Am 1999;105:2287-93.

[10] Van Renterghem T, Botteldooren D, Cornelis W, Gabriels D. Reducing screen-induced refraction of noise barriers in wind with vegetative screens. Acta Acust Acust 2002;88:231-8.

[11] Van Renterghem T, Botteldooren D. Addition to: Reducing screen-induced refraction of noise barriers in wind with vegetative screens. Acta Acust Acust 2003;89:381. 